\title{
Nanoscale
}

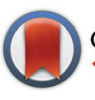

CrossMark

Cite this: Nanoscale, 2015, 7, 12606

\section{Surface enhanced Raman scattering by graphene- nanosheet-gapped plasmonic nanoparticle arrays for multiplexed DNA detection $\uparrow$}

\author{
Bo Duan, ${ }^{a}$ Jiajing Zhou, ${ }^{a}$ Zheng Fang, ${ }^{a}$ Chenxu Wang, ${ }^{a}$ Xiujuan Wang, ${ }^{a}$ \\ Harold F. Hemond, ${ }^{* b}$ Mary B. Chan-Park ${ }^{\star a}$ and Hongwei Duan ${ }^{\star a}$
}

We have developed a new type of surface enhanced Raman scattering (SERS) substrate with thiolated graphene oxide (tGO) nanosheets sandwiched between two layers of closely packed plasmonic nanoparticles. The trilayered substrate is built up through alternative loading of interfacially assembled plasmonic nanoparticle arrays and tGO nanosheets, followed by coating the nanoparticle surfaces with poly(ethylene glycol) (PEG). Here tGO plays multifunctional roles as a 2D scaffold to immobilized interfacially assembled plasmonic nanoparticles, a nanospacer to create SERS-active nanogaps between two layers of nanoparticle arrays, and a molecule harvester to enrich molecules of interest via $\pi-\pi$ interaction. In particular, the molecule harvesting capability of the tGO nanospacer and the stealth properties of PEG coating on the plasmonic nanoparticles collectively lead to preferential positioning of selective targets such as aromatic molecules and single-stranded DNA at the SERS-active nanogap hotspots. We have demonstrated that an SERS assay based on the PEGylated trilayered substrate, in combination with magnetic separation, allows for sensitive, multiplexed "signal-off" detection of DNA sequences of bacterial pathogens.

Received 4th April 2015

Accepted 10th June 2015

DOI: $10.1039 /$ c5nr02164b

www.rsc.org/nanoscale
Ordered arrays of plasmonic nanoparticles can be readily obtained using lithographic patterning techniques. However, it remains challenging to produce large areas of nanoparticle arrays containing exceedingly small nanogaps below $5 \mathrm{~nm}$, which are most efficient in SERS signal amplification. ${ }^{16,17}$ In this context, self-assembly of structure-tailored nanoparticles that allows for scalable production of closely packed nanoparticle ensembles has attracted increasing attention. ${ }^{14,18-23}$ Notably, although considerable success has been achieved in developing SERS substrates with abundant hotspots, ${ }^{24}$ most SERS substrates reported previously lack a mechanism to selectively position the analytes of interest in the SERS hotspots for maximum detection sensitivity.

Here we report a self-assembly approach toward a new class of trilayered SERS substrates with single layer graphene nanosheets sandwiched between two-dimensional (2D) arrays of closely packed plasmonic nanoparticles. Single-atom-thick graphene nanosheets exhibit intriguing optical, electronic, and structural properties. ${ }^{25,26}$ Hybrid materials of structurally integrated graphene derivatives and metal nanoparticles are under intense research for collective optical ${ }^{27,28}$ and catalytic ${ }^{29}$ properties. In our design, graphene oxide (GO) nanosheets play multifunctional roles as a $2 \mathrm{D}$ scaffold to anchor plasmonic nanoparticles through a metal-sulfur bond, a nanometerthick spacer to create SERS-active nanogaps between two layers 


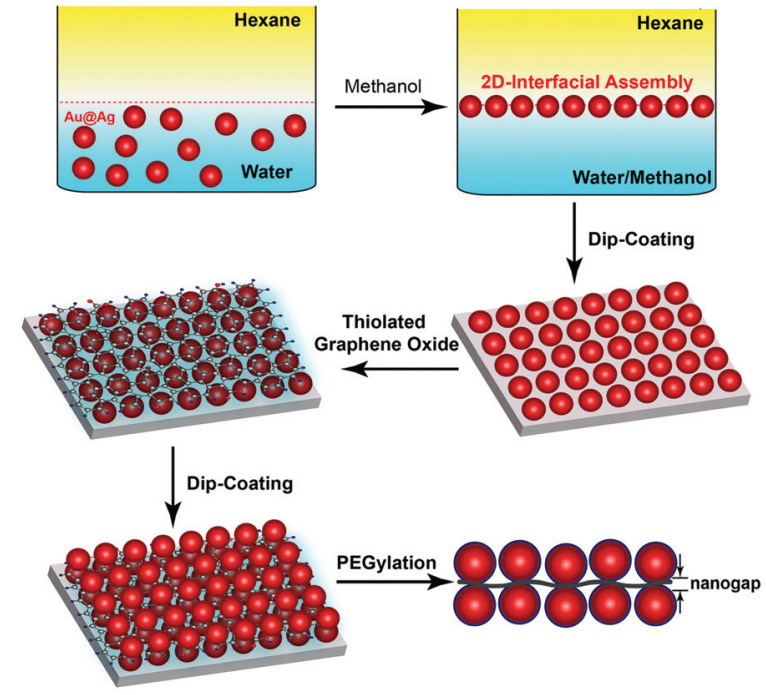

Fig. 1 Schematic illustration of the preparation of a trilayered Au@AgtGO-Au@Ag SERS substrate based on alternative loading of interfacially assembled Au@Ag nanoparticles and thiolated graphene oxide, followed by PEGylation of Au@Ag nanoparticles.

of nanoparticle arrays, and a molecule harvester for targeted analytes by virtue of $\pi-\pi$ interaction. As illustrated in Fig. 1, the trilayered construct is built up through alternative loading of interfacially assembled arrays of Au@Ag core-shell nanoparticles and thiolated graphene oxide (tGO) nanosheets, followed by coating the nanoparticles with poly(ethylene glycol) (PEG) to minimize adsorption of targeted analytes on their surfaces. The nanogap created between the two layers of nanoparticle arrays and molecule harvesting properties of the tGO nanospacer, in conjugation with the stealth PEG coating on plasmonic nanoparticles, collectively leads to precise positioning of selective analytes such as aromatic molecules ${ }^{30}$ and single-stranded DNA (ssDNA) ${ }^{31}$ at the nanogap hotspots for ultrasensitive SERS detection.

Self-assembly of nanoparticles at oil-water interfaces, thermodynamically driven by favorable interfacial energy, has emerged as a promising approach for fabricating large areas of closely attached functional nanocrystals of diverse chemical composition and structures, with interparticle distances tailorable by surface coatings and solvent systems. ${ }^{32-35}$ Recently, the resulting $2 \mathrm{D}$ arrays of functional nanocrystals at oil-water interfaces have found potential uses in SERS, ${ }^{18,20}$ electrochemical biosensors, ${ }^{36}$ and chemical catalysis. ${ }^{37}$ Nevertheless, in contrast to our trilayered substrate with a built-in target enrichment mechanism imparted by the tGO nanospacer, the existing SERS substrates based on a single layer of interfacially assembled plasmonic nanostructures, either freestanding at oil-water interfaces ${ }^{38,39}$ or anchored on solid substrates, ${ }^{40}$ rely primarily on random adsorption of molecules on the nanostructures rather than preferential accumulation in the hotspot region.

Ultrasensitive, multiplex detection of DNA sequences is of considerable interest for both fundamental molecular biology research and practical uses in a wide range of fields such as

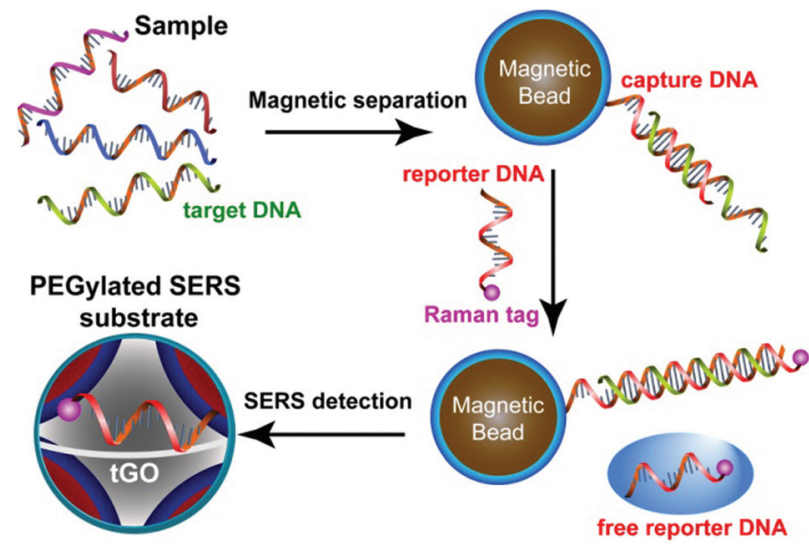

Fig. 2 Schematic illustration of the SERS detection of DNA based on the trilayered GO-gapped plasmonic arrays of Au@Ag core-shell nanoparticles.

diagnostics, food safety, and forensics. Major efforts have been made to develop DNA SERS sensors based on DNA-functionalized plasmonic nanostructures. ${ }^{38-40}$ Previous research has demonstrated that exposed nucleobases of flexible ssDNA endow them with strong affinity to GO nanosheets due to $\pi-\pi$ interaction. ${ }^{31,41,42}$ This affinity allows us to develop a "signaloff” DNA sensor using our trilayered SERS substrate. Two unique oligonucleotide strands containing part of the complementary sequence of the target DNA are linked to magnetic beads (MB) and a Raman dye and serve as capture and reporter DNA, respectively. In a sandwich assay (Fig. 2), MB-bound capture DNA first hybridizes with the target DNA to separate and concentrate it from the sample under test, and the overhanging region of the target DNA is then recognized by the Raman-encoded reporter DNA. In the absence of the targeted DNA, the reporter DNAs are not removed and all bind to the GO nanospacer, leading to the strongest SERS signal. However, when the targeted DNA is present to hybridize with the capture and reporter DNA, Raman tags on the reporter DNA are not able to locate inside the nanogap hotspots after hybridization, which turns off the SERS signal. In addition to the extraordinary signal amplification of SERS spectroscopy, the structure-defined spectral fingerprints of individual Raman tags and narrow bandwidth of Raman peaks, along with single-wavelength excitation of multiple tags, collectively provide unique opportunities for multiplexed detection. Here, using the DNA sequences of three food-borne pathogens as model targets, we have shown that our tGO-gapped plasmonic substrates enable highly efficient multiplexed DNA detection down to a concentration of $10 \mathrm{pM}$.

\section{Results and discussion}

GO nanosheets prepared by the modified Hummers method carry a high density of oxygen-containing groups, primarily hydroxyl and epoxy groups in the basal plane and carboxylic 
acid groups at the edges, which have low affinity to metal surfaces. To function as a $2 \mathrm{D}$ anchoring scaffold for stable loading of metal nanostructures, new functionalities with stronger binding to metal surfaces are necessitated. Here, we have introduced thiol groups, which can form metal-sulfur bonds, by taking advantage of the ring-opening reaction of epoxy groups on the basal plane of GO and the primary amine group of cysteine. ${ }^{43,44}$ X-ray photoelectron spectroscopy (XPS) measurements (Fig. 3a) show that a new peak $(167.0 \mathrm{eV})$ associated with S 2p level binding energy appears in the modified tGO, confirming the successful functionalization. In the deconvolved C 1s peaks of GO and tGO (Fig. 3b), the weaker peak $(284.6 \mathrm{eV})$ of $\mathrm{sp}^{2}$-bonded carbon in GO becomes the dominant peak in tGO, in comparison with the epoxy/hydroxyl $(286.9 \mathrm{eV})$ and carbonyl $(288.5 \mathrm{eV})$ peaks, suggesting that cysteine in large excess also leads to partial reduction of GO. Recent reports have shown that reductive thiol-containing molecules such as glutathione can effectively reduce GO. ${ }^{45}$ In addition, Raman spectra (Fig. 3c) display an increased intensity ratio of the $\mathrm{D}$ and $\mathrm{G}$ bands after the reaction, which is consistent with the partial restoration of the $\mathrm{sp}^{2}$ carbon network in tGO. It should be mentioned that tGO maintains excellent colloidal stability in aqueous media and exists as individual nanosheets, which is essential for its use as a nanospacer in the trilayered SERS substrate. Atomic force microscopy (AFM) measurements (Fig. 3d) reveal that the nanosheets have a representative thickness of about $1.2 \mathrm{~nm}$, which is consistent with the thickness of a single layer of chemically derived GO.

We synthesized core-shell Au@Ag nanoparticles of $30 \mathrm{~nm}$ through controlled seeded growth of an $\mathrm{Ag}$ shell on citratestabilized Au nanoparticles of $15 \mathrm{~nm}$ in diameter. Addition of
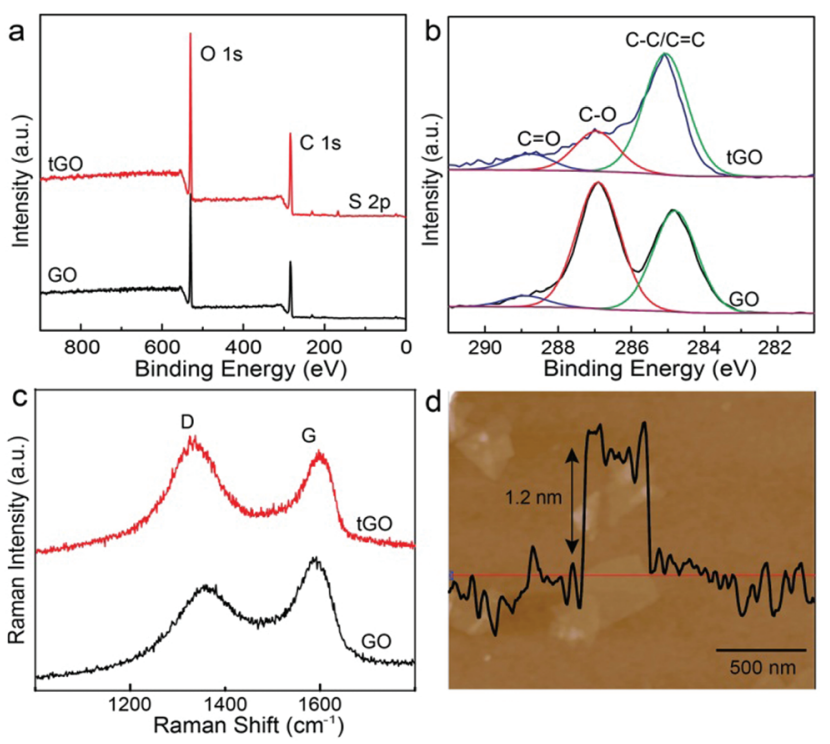

Fig. 3 (a) XPS spectra of graphene oxide (GO) and cysteine functionalized graphene oxide (tGO). (b) The deconvolved $C$ 1s spectra of $G O$ and tGO. (c) Raman spectra of GO and tGO. (d) AFM image and height profile of tGO.
$\mathrm{AgNO}_{3}$ solution to $\mathrm{Au}$ nanoparticle dispersion containing L-ascorbic acid quickly led to a color change from red to bright yellow and LSPR of the nanoparticles shifted from $520 \mathrm{~nm}$ to $400 \mathrm{~nm}$ (Fig. 4a). Transmission electron microscopy (TEM) observation (Fig. $\mathrm{S} 1 \dagger$ ) reveals that a $7 \mathrm{~nm} \mathrm{Ag}$ layer with lower contrast formed on the Au seeds, leading to core-shell Au@Ag nanoparticles of $\sim 30 \mathrm{~nm}$ in size. Previous reports have shown that the $\mathrm{Ag}$ shell dominates the optical properties of $\mathrm{Au} @ \mathrm{Ag}$ nanoparticles $^{46}$ and Au@Ag nanoparticles exhibit Raman enhancement efficiencies comparable to those of pure $\mathrm{Ag}$ nanoparticles. ${ }^{47-49}$

The tendency of nanoparticles to accumulate at oil-water interfaces is closely related to the size of the nanoparticles, interfacial energy of the biphasic system, and contact angle of the nanoparticles at the interface, where a contact angle of $90^{\circ}$ provides the largest free energy change. ${ }^{33}$ It has been reported that adding alcohol to a biphasic mixture of aqueous dispersion of charged particles and organic solvents reduces the charge density on the particles and consequently tunes their contact angle at the oil-water interface close to $90^{\circ}$, giving rise to $2 \mathrm{D}$-assemblies of the particles at the interface. ${ }^{34,35}$ Here, methanol was added into the mixture of aqueous dispersion of $\mathrm{Au} @ A g$ nanoparticles and hexane to trigger their interfacial assembly. As shown in the inset of Fig. 4a, Au@Ag nanoparticles moved to the interface and formed a layer exhibiting metallic reflectance upon the addition of methanol followed by vigorous shaking to facilitate the migration of the nanoparticles. The LSPR peak of Au@Ag nanoparticles experienced a drastic red-shift from $400 \mathrm{~nm}$ to $700 \mathrm{~nm}$, indicative of a
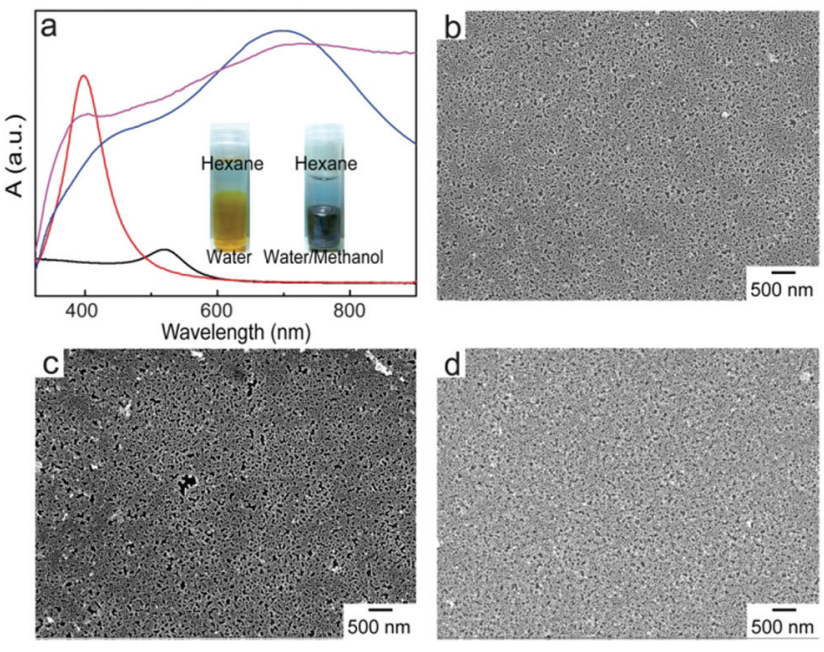

Fig. 4 (a) UV-Vis spectra of Au nanoparticle dispersion (black line), AuaAg nanoparticle dispersion (red line), 2D assembly of Au@Ag nanoparticles (blue line), and the Au@Ag-tGO-Au@Ag trilayered construct (purple line). The spectra have been offset vertically for better comparison of absorption maxima. The insets are photographs of Au@Ag nanoparticle dispersion (left) and the interfacially assembled nanoparticles (right). (b-d) SEM images of 2D assembly of AuaAg nanoparticles (b), bilayered Au@Ag-tGO structure (c) and Au@Ag-tGO-Au@Ag sandwich structure (d) deposited on glass substrates. 
strong interparticle plasmonic coupling. The interfacial assembly was transferred to a glass substrate, which was pretreated with (3-mercaptopropyl)trimethoxysilane. The pretreatment was to introduce thiol groups on the substrate for stable anchoring of the nanoparticles. Without it, the loaded nanoparticles were quickly washed off once they were in contact with the aqueous medium. A monolayer of closely packed nanoparticles was observed in the scanning electron microscopy (SEM) image (Fig. 4b). The coated substrate was further immersed in the tGO aqueous dispersion to introduce the tGO nanospacer on top of the nanoparticle monolayer (Fig. 4c). The presence of a homogeneous layer of $\mathrm{tGO}$ was confirmed by the consistent Raman signal of chemically derived GO across the entire substrate of a few centimeters in lateral size (Fig. S2 $\dagger$ ). In contrast, unmodified GO without the thiol groups cannot form stable loading on the nanoparticle array, evidenced by the absence of Raman signals from GO. Afterwards, the second layer of Au@Ag nanoparticles was added by dip-coating to form the sandwiched Au@Ag-tGO-Au@Ag trilayered construct, as shown in Fig. 4d. The binding of thiol functional groups onto the tGO basal plane with nanoparticle surfaces aligns the $2 \mathrm{D}$ anchoring scaffold parallel to the substrate, which in turn leads to the formation of nanogaps between the bottom and top nanoparticle monolayers. The close attachment of two layers of Au@Ag nanoparticles gives rise to further red shift and broadening of the LSPR peak. PEG chains (molecular weight $=750 \mathrm{Da})$ with one primary amine end group were coated onto the Au@Ag nanoparticles by ligand exchange. Notably, sandwiching tGO between the two layers of Au@Ag nanoparticle arrays to form the nanogap gave rise to an increase of the tGO Raman signal by a factor of 8 (Fig. S2 $\dagger$ ). SEM observations confirm that the PEGylated nanoparticle arrays did not undergo structural changes and remained stable for at least 3 months (Fig. S3a †). In contrast, without PEGylation, the nanoparticles apparently fuse together to form large clusters within $48 \mathrm{~h}$ after preparation (Fig. S3b †े).

We next evaluated the SERS activity of the PEGylated Au@Ag-tGO-Au@Ag substrate using Rhodamine B (RhB) as a model Raman probe. Both the tGO-gapped architecture and PEGylation coating appear to make major contributions to the superior SERS activity of the substrate. RhB is a commonly used Raman dye molecule that adsorbs on citrate-stabilized $\mathrm{Au} @ \mathrm{Ag}$ nanoparticles primarily through electrostatic interactions. Our results demonstrate that the PEG coating grafted on colloidal Au@Ag nanoparticles can effectively suppress the non-specific adsorption of RhB molecules on the SERS-active sites of their surfaces (Fig. S4 $\dagger$ ). We reason that a dense layer of PEG chains either forms a physical barrier to block the access of $\mathrm{RhB}$ molecules to the nanoparticle surfaces or occupies the SERS-active sites.

When the Au@Ag nanoparticle surfaces are PEGylated, the trilayered Au@Ag-tGO-Au@Ag structure exhibits a dramatic signal enhancement by a factor of 20 relative to that of the bilayered Au@Ag-tGO substrates for the RhB concentration of $100 \mathrm{nM}$, as shown in Fig. 5a. Graphene derivatives with a sp ${ }^{2}$ hybridized carbon network have been widely employed for
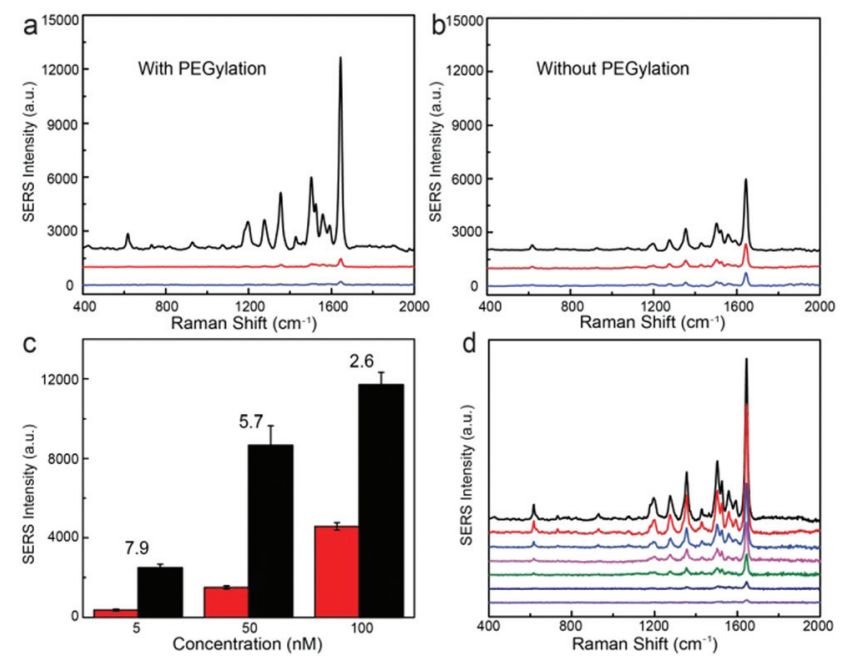

Fig. 5 (a and b) SERS spectra of RhB (100 nM) on (a) PEGylated and (b) non-PEGylated substrates: Au@Ag NP monolayered (blue line), Au@AgtGO bilayered structure (red line), and $A u(A g-t G O-A u @ A g$ sandwich type substrate (black line). (c) SERS intensity of RhB on AuaAg-tGOAu@Ag with (black) and without (red) PEGylation as a function of RhB concentration. The numbers represent the factor of SERS intensity increase after PEGylation. (d) SERS spectra of RhB on PEGylated Au@Ag-tGO-Au@Ag substrate at different concentrations, from bottom to top: $0.05,0.5,1,5,10,50$, and $100 \mathrm{nM} \mathrm{RhB}$.

loading aromatic molecules through $\pi-\pi$ interactions. In a PEGylated trilayered substrate, this molecule harvesting property of tGO gives rise to preferential enrichment of RhB molecules in the SERS hotspot between the two layers of Au@Ag NP arrays, thereby resulting in considerably improved SERS activity with respect to that of the PEGylated Au@Ag-tGO bilayered substrate, in which $\mathrm{tGO}$ nanosheets are placed on top of Au@Ag NPs instead of in the nanogap SERS hotspots. We have found that PEGylation of the nanoparticle surfaces in the trilayered construct gives rise to a marked increase in the SERS signal (black lines in Fig. 5a and b) and the degree of increase becomes more prominent at low concentrations of analytes (Fig. 5c). In contrast, PEGylation of the nanoparticles in both the bilayered Au@Ag-tGO and monolayered Au@Ag substrates led to an obvious signal decrease. To avoid interference caused by nanoparticle fusion, freshly prepared nonPEGylated substrates were used in the SERS measurements for comparison. The closely packed Au@Ag NP array contains highly SERS-active interstitial spaces and less active superficial surfaces. As discussed above, PEGylation of Au@Ag NPs deactivates the nanoparticle surfaces for capturing RhB probes, and consequently leads to reduced SERS activity for PEGylated monolayered and bilayered substrates (Fig. $5 \mathrm{a}$ and b). For the trilayered substrate, however, the deactivation of Au@Ag nanoparticle surfaces by PEGylation minimizes the binding of RhB molecules to the less SERS-active superficial surfaces of nanoparticles, which would otherwise occur in a non-PEGylated substrate, giving rise to selective accumulation of RhB molecules on the tGO nanospacer where the SERS hotspots are 
located. The enhancement induced by PEGylation, defined as the factor of SERS intensity increase after PEGylation, became more pronounced at a low $\mathrm{RhB}$ concentration and increased from 2.6 at $100 \mathrm{nM}$ to 7.9 at $5 \mathrm{nM}$ (Fig. 5c), demonstrating the importance of selectively positioning molecules in the SERS hotspots for ultrasensitive detection. The trilayered PEGylated $\mathrm{Au} @ \mathrm{Ag}-\mathrm{tGO}-\mathrm{Au} @ \mathrm{Ag}$ substrate gives rise to sensitive detection of RhB (Fig. 5d) and a SERS enhancement factor of $2.7 \times 10^{8}$ (see the ESI $\dagger$ ). Although recent studies have demonstrated that graphene has SERS activity, leading to an enhancement factor of 2-17 through a chemical effect, the contribution of electromagnetic enhancement plays a dominant role in the PEGylated $\mathrm{Au} @ \mathrm{Ag}-\mathrm{tGO}-\mathrm{Au} @ \mathrm{Ag}$ substrate, considering the enhancement factor it exhibits.

The PEGylated trilayered substrate was further utilized for the detection of gene sequences of bacterial pathogens such as E. coli $\mathrm{O} 157: \mathrm{H7}$, Staphylococcus aureus, and Listeria monocytogenes, which are food-borne pathogens of significant public health risks. Sequence information on the DNA used in this work is summarized in Table S1.† Ultrasensitive detection of DNA sequences relies on the selective enrichment of reporter DNA in the nanogap hotspots. We have found that fluorescence quenching of TAMRA tags on SsDNA by Au@Ag nanoparticles decreased from $84 \%$ to $9 \%$ (Fig. S5†) after PEGylation, confirming that PEG grafts can effectively prevent adsorption of ssDNA onto nanoparticle surfaces. ${ }^{50}$ We further examined SERS detection of HEX-labeled reporter DNA using the PEGylated Au@Ag-tGO-Au@Ag substrate. Fig. 6a shows that the SERS signal of the HEX dye at $1626 \mathrm{~cm}^{-1}$ gradually increased in the DNA concentration range of $2-100 \mathrm{nM}$ and eventually leveled off. A typical sandwich assay involves three steps: first, the target DNA of E. coli $\mathrm{O} 157: \mathrm{H} 7$ in $1 \mathrm{~mL}$ of sample solution is recognized by the MB-bound capture DNA and magnetically enriched; second, a predetermined amount $(20 \mu \mathrm{L}, 20 \mathrm{nM})$ of HEX-tagged reporter DNA is then introduced to hybridize with the overhanging region of the concentrated target DNA; finally, the sandwich structure formed on MB is magnetically separated and the remaining free reporter DNA is subjected to SERS measurement. Fig. 6b shows that the target DNA with an original concentration of $1.0 \mathrm{nM}$ caused an $80 \%$ signal drop for reporter DNA of $20 \mathrm{nM}$. In contrast, scramble non-matching DNA gave rise to minimal signal changes of less than $10 \%$, which could result from non-specific removal of the reporter DNA. This setup allowed for sensitive "signal-off" detection of DNA sequences down to 10 pM (Fig. 6c). Furthermore, the detection system also showed excellent specificity. As demonstrated in Fig. 6d, while the increase of target DNA concentration from $10 \mathrm{pM}$ to $1.6 \mathrm{nM}$ led to continuous signal drop, the SERS signal remained consistently unchanged in the presence of non-complementary DNA of the same concentration.

Simultaneous detection of multiple DNA sequences is of particular importance in rapid identification of pathogens. A large group of Raman dyes with distinct spectral profiles are readily available for tagging ssDNA, opening the possibility for multiplexed DNA detection. Here, HEX, Cy5, and TAMRA were
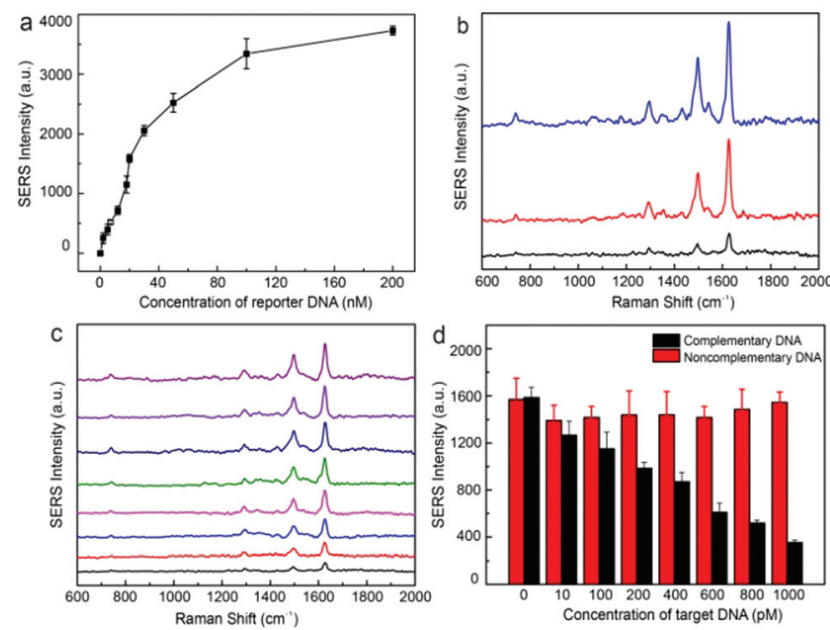

Fig. 6 (a) The dependence of SERS signal intensity at $1626 \mathrm{~cm}^{-1}$ on the concentration of HEX-labeled reporter DNA on PEGylated AuAAgtGO-Au@Ag trilayered substrate. (b) SERS spectra of HEX-labeled reporter DNA in the absence and presence of target DNA, and in the presence of a non-complementary sequence (blue trace: HEX labeled reporter DNA $(20 \mathrm{nM})$, red trace: HEX-labeled reporter DNA incubated in the presence of a non-complementary DNA sequence, and black trace: HEX labeled reporter DNA for the detection of $1.0 \mathrm{nM}$ complementary target DNA). (c) SERS spectra of HEX-labeled reporter DNA (20 nM) for the detection of target DNA of different concentrations, from top to bottom: $0,0.01,0.1,0.2,0.4,0.6,0.8,1.0 \mathrm{nM}$. (d) Comparison of SERS intensity of HEX-labeled reporter DNA at $1626 \mathrm{~cm}^{-1}$ for the detection of target DNA (black) and non-complementary DNA (red) of various concentrations.

used to encode the reporter DNAs for the detection of gene sequences of E. coli $\mathrm{O} 157: \mathrm{H7}$, Staphylococcus aureus, and Listeria monocytogenes, respectively. The SERS spectra of Cy5 and TAMRA labeled reporter DNA are shown in Fig. S6 and S7. $\dagger$ The Raman peaks at 1626, 1465/1594, and $1645 \mathrm{~cm}^{-1}$ were selected as the characteristic peaks for HEX, ${ }^{52} \mathrm{Cy} 5,{ }^{38}$ and TAMRA $^{51}$ tags, respectively. Different concentrations of reporter DNAs (20 nM for HEX, $15 \mathrm{nM}$ for TAMRA, and $50 \mathrm{nM}$ for Cy5) were mixed to match their differences in Raman crosssection, leading to a spectrum containing combined Raman peaks of all three dyes (Fig. 7a). In representative measurements, addition of selected target DNA of $1.0 \mathrm{nM}$ into the mixture specifically turned off the corresponding Raman peaks (Fig. 7b-d), demonstrating the multiplexed capability of our SERS sensor.

\section{Experimental section}

\subsection{Materials}

Graphite, hydrogen tetrachloroauric acid $\left(\mathrm{HAuCl}_{4} \cdot 3 \mathrm{H}_{2} \mathrm{O}\right)$, silver nitrate, trisodium citrate, hexane, methanol, nitric acid, hydrochloric acid, sulphuric acid, hydrogen peroxide, (3-mercaptopropyl)trimethoxysilane (MPTMS), methoxypolyethylene glycol amine (PEG-NH $\left.\mathrm{N}_{2}, M_{\mathrm{W}}=750 \mathrm{Da}\right)$ and L-cysteine were purchased from Sigma-Aldrich and used without purification. Rhodamine 


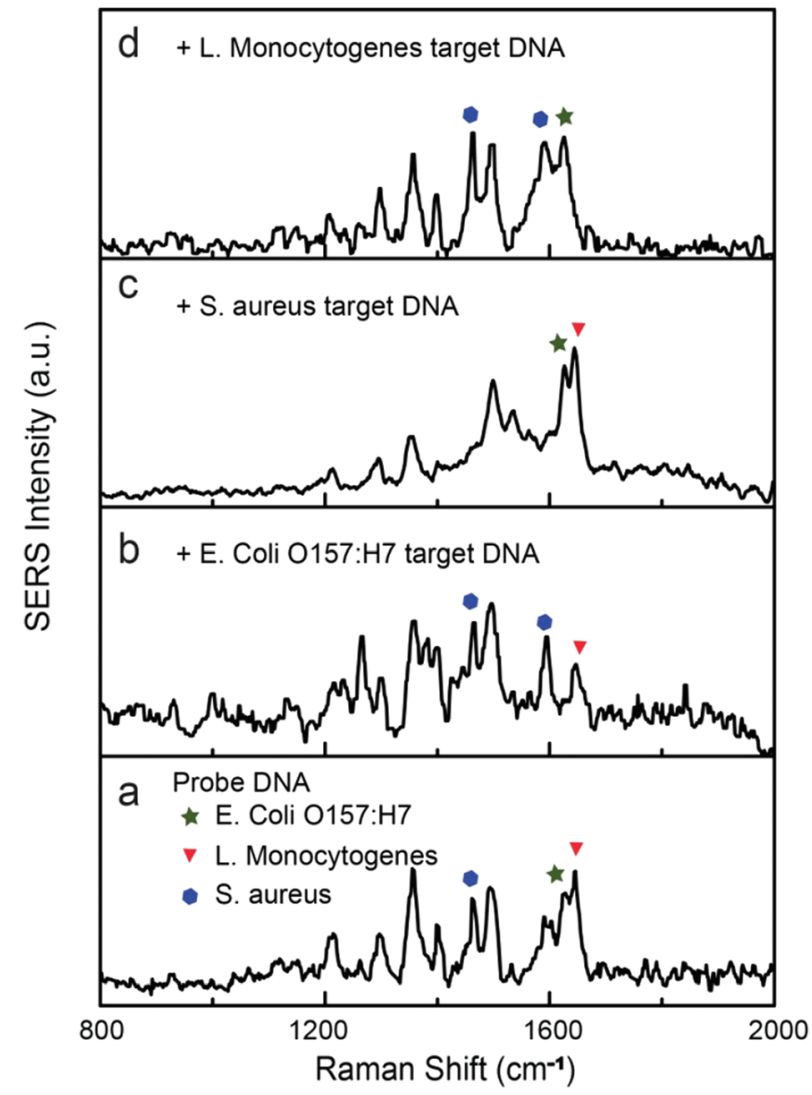

Fig. 7 (a) SERS spectra of a mixture of reporter DNAs (20 nM for HEX, $15 \mathrm{nM}$ for TAMRA, and $50 \mathrm{nM}$ for Cy5). (b-d) SERS spectra collected for the detection of target DNAs (1.0 nM) of (b) E. coli O157:H7, (c) Staphylococcus aureus, and (d) Listeria monocytogenes. Triangle, star, and hexagon respectively indicate characteristic Raman peaks of TARMA, $\mathrm{HEX}$, and Cy5, respectively.

B was acquired from Sigma-Aldrich at $>90 \%$ purity. ssDNAs were purchased from Shanghai Sangon Biotechnology Incorporation (Shanghai, China). Water used in this experiment was deionized water (DI water). Glassware used for all experiments were cleaned using aqua regia $\left(\mathrm{HCl} / \mathrm{HNO}_{3} 3: 1(\mathrm{v} / \mathrm{v})\right)$ and rinsed with copious amounts of DI water prior to use.

\subsection{Functionalization of graphene oxide (GO)}

Graphene oxide (GO) was synthesized using the modified Hummers method ${ }^{53,54}$ by oxidation of graphite powder. Synthesized GO nanosheets were purified via multiple centrifugations and then re-dispersed in water to form a well-dispersed suspension. Further functionalization of GO nanosheets with L-cysteine was carried out following our previously reported method. $^{44}$

\subsection{Au@Ag nanoparticle preparation}

Au@Ag nanoparticles with a diameter of $30 \mathrm{~nm}$ were synthesized by a seed-mediated method using $15 \mathrm{~nm}$ Au nanoparticles as seeds. $15 \mathrm{~nm} \mathrm{Au}$ nanoparticles were prepared by direct reduction of $\mathrm{HAuCl}_{4}$ using sodium citrate as both a reducing agent and a capping agent. In a typical synthesis, $\mathrm{HAuCl}_{4}(60 \mathrm{mg})$ was dissolved in DI water $(400 \mathrm{~mL})$ and heated to boiling. Upon boiling, a solution of trisodium citrate tetrahydrate (200 mg, $4.5 \mathrm{~mL}$ ) was quickly injected. Heating was maintained for another 30 minutes, and the resultant wine-red solution was cooled to room temperature. To synthesize $30 \mathrm{~nm}$ core-shell nanoparticles, $2 \mathrm{~mL}$ of as-prepared $15 \mathrm{~nm}$ Au seed nanoparticles was first diluted with $30 \mathrm{~mL}$ of DI water, then L-ascorbic acid (10 $\mathrm{mg} \mathrm{mL}^{-1}, 10 \mathrm{~mL}$ ) was added, followed by the slow addition of silver nitrate $\left(0.2 \mathrm{mg} \mathrm{mL}^{-1}, 5 \mathrm{ml}\right)$.

\subsection{Fabrication of Au@Ag-tGO-Au@Ag sandwich structured substrates}

Glass substrates were first cleaned by sonication in ethanol and water for 10 minutes each. To make the surface hydrophilic, cleaned glass substrates were treated with piranha solution $\left(3: 1 \mathrm{v} / \mathrm{v} \quad \mathrm{H}_{2} \mathrm{SO}_{4} \quad(98 \%) / \mathrm{H}_{2} \mathrm{O}_{2} \quad(30 \%)\right)$ at $80{ }^{\circ} \mathrm{C}$ for 30 minutes. After rinsing with copious amounts of water and ethanol, the glass substrates were dried thoroughly and then immersed in 5 mM MPTMS solution for 30 minutes for silanization. Silane modified glass substrates were then rinsed thoroughly with chloroform and water to remove excess MPTMS. To prepare self-assembled Au@Ag NP film, hexane $(0.5 \mathrm{~mL})$ was added into the aqueous suspension of $\mathrm{Au} @ \mathrm{Ag}$ NPs $(1 \mathrm{~mL})$, followed by slow addition of methanol $(0.1 \mathrm{~mL})$. $\mathrm{Au} @ A g$ nanoparticles were spontaneously transferred to the water/hexane interface and self-assembled into a single layer film. This film formation process could be accelerated by shaking. Self-assembled Au@Ag nanoparticle film was then transferred onto silane-modified glass substrates and dried in ambient air. Subsequent immersion in tGO dispersion for 4 hours successfully yielded a tGO-coated substrate. Lastly, a second layer of Au@Ag nanoparticle film was prepared and transferred onto the glass substrate, resulting in a substrate with tGO nanosheets sandwiched between two monolayers of $\mathrm{Au} @ A g$ nanoparticle assemblies. PEGylation was performed by soaking the substrates in PEG- $\mathrm{NH}_{2}$ solution for 4 hours.

\subsection{Synthesis of magnetic beads and DNA conjugation}

Polydopamine (PDA) coated magnetic beads (MB@PDA) were prepared by in situ growth of polydopamine on magnetic beads in $\mathrm{pH} 8.5$ tris buffer through self-polymerization of dopamine. Thiol-functionalized capture DNA was attached on MB@PDA colloid via the Michael addition reaction at a salt concentration of $80 \mathrm{mM}$ for $8 \mathrm{~h}$, followed by PEGylation with PEG-NH ${ }_{2}\left(M_{\mathrm{W}}=2000 \mathrm{Da}\right)$. MB-DNA conjugates were purified by an external magnetic field and then mixed with test solutions containing the target DNA. After incubation for $4 \mathrm{~h}$ at room temperature, the solid was separated by a magnetic field and redispersed in buffer $(10 \mathrm{mM}$ phosphate, $\mathrm{pH}=7.4)$ solution containing $80 \mathrm{mM} \mathrm{NaCl}$. MBs carrying the target DNA were then subjected to Raman-dye-tagged reporter DNA and incubated for another $4 \mathrm{~h}$. The solid was again separated by a magnetic field and the solution containing the free reporter DNA that was left after hybridization was further introduced 
into the Au@Ag-tGO-Au@Ag substrate and incubated for $2 \mathrm{~h}$ before measurement.

\subsection{Characterization and measurements}

UV-visible absorption spectra were recorded using a Shimadzu UV2501 spectrophotometer. Scanning electron microscopy (SEM) images were obtained using a field-emission scanning electron microscope (JSM-6700F). Raman spectra were acquired using a Renishaw Raman microscope. The $514 \mathrm{~nm}$ excitation line from an air-cooled $\operatorname{Ar}(+)$ laser was used for both normal Raman and SERS measurements. The laser beam has a spot size of $1 \mu \mathrm{m}$ using a $50 \times$ objective. A single scan with an integration time of $10 \mathrm{~s}$ was performed for each spectrum acquisition. X-ray photoelectron spectroscopy (XPS) measurement was carried out on a Kratos-Axis spectrometer with monochromatic $\mathrm{Al} \mathrm{K} \alpha(1486.7 \mathrm{eV}) \mathrm{X}$-ray radiation $(15 \mathrm{kV}$ and $10 \mathrm{~mA}$ ) and a hemispherical electron energy analyzer.

\section{Conclusions}

In summary, we have developed a modular approach for fabricating efficient SERS substrates based on the use of chemically derived GO and interfacial assembly of plasmonic nanoparticles as building blocks. The multifunctional role of tGO as a $2 \mathrm{D}$ scaffold, a nanospacer and a molecule harvester makes it possible to selectively position the targeted analytes in the SERS hotspots. The modular nature of our strategy and recent advances in tailored wet-chemical synthesis of plasmonic nanocrystals collectively provide new opportunities for achieving fundamental insights into the architecture-SERS performance relationship. Furthermore, the ability of graphene derivatives to bind SsDNA, coupled with the powerful multiplexing of SERS spectroscopy, empowers this SERS substrate for ultrasensitive multiplexed genetic detection of pathogens.

\section{Acknowledgements}

The authors thank Singapore Ministry of Education for financial support (Tier 1 project RGT19/13 and Tier 3 project MOE2013-T3-1-002). This research was also supported in part by the National Research Foundation Singapore through the Singapore MIT Alliance for Research and Technology's Center for Environmental Sensing and Modeling interdisciplinary research program.

\section{Notes and references}

1 S. M. Nie and S. R. Emory, Science, 1997, 1102-1106.

2 K. Kneipp, Y. Wang, H. Kneipp, L. T. Perelman, I. Itzkan, R. R. Dasari and M. S. Feld, Phys. Rev. Lett., 1997, 78, 16671670.

3 W. Xie and S. Schlucker, Phys. Chem. Chem. Phys., 2013, 15, 5329-5344.
4 J. Song, J. Zhou and H. Duan, J. Am. Chem. Soc., 2012, 134, 13458-13469.

5 D. A. Stuart, J. M. Yuen, N. Shah, O. Lyandres, C. R. Yonzon, M. R. Glucksberg, J. T. Walsh and R. P. Van Duyne, Anal. Chem., 2006, 78, 7211-7215.

6 B. Peng, G. Li, D. Li, S. Dodson, Q. Zhang, J. Zhang, Y. H. Lee, H. V. Demir, X. Yi Ling and Q. Xiong, ACS Nano, 2013, 7, 5883-6000.

7 M. Lin, Handbook of Vibrational Spectroscopy, John Wiley \& Sons, Ltd, 2006.

8 I. López-Tocón, J. C. Otero, J. F. Arenas, J. V. Garcia-Ramos and S. Sanchez-Cortes, Anal. Chem., 2011, 83, 2518-2525.

9 J. Yin, T. Wu, J. Song, Q. Zhang, S. Liu, R. Xu and H. Duan, Chem. Mater., 2011, 23, 4756-4764.

10 R. A. Alvarez-Puebla and L. M. Liz-Marzán, Small, 2010, 6, 604-610.

11 J. P. Camden, J. A. Dieringer, J. Zhao and R. P. Van Duyne, Acc. Chem. Res., 2008, 41, 1653-1661.

12 J. D. Caldwell, O. Glembocki, F. J. Bezares, N. D. Bassim, R. W. Rendell, M. Feygelson, M. Ukaegbu, R. Kasica, L. Shirey and C. Hosten, ACS Nano, 2011, 5, 4046-4055.

13 J. W. Liu, S. Y. Zhang, H. Qi, W. C. Wen and S. H. Yu, Small, 2012, 8, 2412-2420.

14 G. Lu, H. Li and H. Zhang, Small, 2012, 8, 1336-1340.

15 B. Yan, A. Thubagere, W. R. Premasiri, L. D. Ziegler, L. Dal Negro and B. r. M. Reinhard, ACS Nano, 2009, 3, 11901202.

16 E. C. Le $\mathrm{Ru}$ and P. G. Etchegoin, Principles of SurfaceEnhanced Raman Spectroscopy, Elsevier, 2009.

17 Y. Zheng, T. Thai, P. Reineck, L. Qiu, Y. Guo and U. Bach, Adv. Funct. Mater., 2013, 23, 1519-1526.

18 M. P. Cecchini, V. A. Turek, J. Paget, A. A. Kornyshev and J. B. Edel, Nat. Mater., 2013, 12, 165-171.

19 M. P. Konrad, A. P. Doherty and S. E. J. Bell, Anal. Chem., 2013, 85, 6783-6789.

20 K. Kim, H. S. Han, I. Choi, C. Lee, S. G. Hong, S.-H. Suh, L. P. Lee and T. Kang, Nat. Commun., 2012, 4, 2182.

21 S. Y. Zhang, J. W. Liu, C. L. Zhang and S. H. Yu, Nanoscale, 2013, 5, 4223-4229.

22 T. Wen and S. A. Majetich, ACS Nano, 2011, 5, 8868-8876.

23 A. Chen, A. E. DePrince, A. Demortière, A. Joshi-Imre, E. V. Shevchenko, S. K. Gray, U. Welp and V. K. VlaskoVlasov, Small, 2011, 7, 2365-2371.

24 D.-K. Lim, K.-S. Jeon, J.-H. Hwang, H. Kim, S. Kwon, Y. D. Suh and J.-M. Nam, Nat. Nanotechnol., 2011, 6, 452460.

25 K. P. Loh, Q. L. Bao, G. Eda and M. Chhowalla, Nat. Chem., 2010, 2, 1015-1024.

26 L. Xie, X. Ling, Y. Fang, J. Zhang and Z. Liu, J. Am. Chem. Soc., 2009, 131, 9890-9891.

27 J. Huang, X. Gao, J. Jia, J.-K. Kim and Z. Li, Anal. Chem., 2014, 86, 3209-3215.

28 X. Liu, L. Cao, W. Song, K. Ai and L. Lu, ACS Appl. Mater. Interfaces, 2011, 3, 2944-2952.

29 S. Zhang, Y. Shao, H.-g. Liao, J. Liu, I. A. Aksay, G. Yin and Y. Lin, Chem. Mater., 2011, 23, 1079-1081. 
30 P. Wang, O. Liang, W. Zhang, T. Schroeder and Y.-H. Xie, Adv. Mater., 2013, 25, 4918-4924.

31 S. He, B. Song, D. Li, C. Zhu, W. Qi, Y. Wen, L. Wang, S. Song, H. Fang and C. A. Fan, Adv. Funct. Mater., 2010, 20, 453-459.

32 L. Cheng, A. P. Liu, S. Peng and H. W. Duan, ACS Nano, 2010, 4, 6098-6104.

33 H. Duan, D. Wang, D. G. Kurth and H. Möhwald, Angew. Chem., Int. Ed., 2004, 43, 5639-5642.

34 Y.-J. Li, W.-J. Huang and S.-G. Sun, Angew. Chem., Int. Ed., 2006, 45, 2537-2539.

35 F. Reincke, S. G. Hickey, W. K. Kegel and D. Vanmaekelbergh, Angew. Chem., Int. Ed., 2004, 43, 458462.

36 F. Xiao, J. Song, H. Gao, X. Zan, R. Xu and H. Duan, ACS Nano, 2011, 6, 100-110.

37 J. Zhou, B. Duan, Z. Fang, J. Song, C. Wang, P. B. Messersmith and H. Duan, Adv. Mater., 2014, 26, 701705.

38 T. Kang, S. M. Yoo, I. Yoon, S. Y. Lee and B. Kim, Nano Lett., 2010, 10, 1189-1193.

39 D.-K. Lim, K.-S. Jeon, H. M. Kim, J.-M. Nam and Y. D. Suh, Nat. Mater., 2010, 9, 60-67.

40 Y. C. Cao, R. Jin and C. A. Mirkin, Science, 2002, 297, 15361540.

41 M. Wu, R. Kempaiah, P.-J. J. Huang, V. Maheshwari and J. Liu, Langmuir, 2011, 27, 2731-2738.
42 C.-H. Lu, H.-H. Yang, C.-L. Zhu, X. Chen and G.-N. Chen, Angew. Chem., Int. Ed., 2009, 48, 4785-4787.

43 Q. Mei and Z. Zhang, Angew. Chem., Int. Ed., 2012, 51, 5602-5606.

44 Z. Fang, Y. Wang, J. Song, Y. Sun, J. Zhou, R. Xu and H. Duan, Nanoscale, 2013, 5, 9830-9838.

45 H. Gao, F. Xiao, C. B. Ching and H. Duan, ACS Appl. Mater. Interfaces, 2012, 4, 2801-2810.

46 S.-I. Ana, C.-A. Enrique, G. Arnaud, R.-G. Benito, P.-J. Jorge, P.-S. Isabel and M. L.-M. Luis, Chem. - Eur. J., 2010, 16, 5558-5563.

47 B. Liu, G. Han, Z. Zhang, R. Liu, C. Jiang, S. Wang and M.-Y. Han, Anal. Chem., 2011, 84, 255-261.

48 X. Yang, C. Gu, F. Qian, Y. Li and J. Z. Zhang, Anal. Chem., 2011, 83, 5888-5894.

49 A. Shen, J. Guo, W. Xie, M. Sun, R. Richards and J. Hu, Raman Spectrosc., 2011, 42, 879-884.

50 H. Li and L. Rothberg, Proc. Natl. Acad. Sci. U. S. A., 2004, 101, 14036-14039.

51 S. H. Yazdi, K. L. Giles and I. M. White, Anal. Chem., 2013, 85, 10605-10611.

52 K. Faulds, W. E. Smith and D. Graham, Anal. Chem., 2003, 76, 412-417.

53 W. S. Hummers and R. E. Offeman, J. Am. Chem. Soc., 1958, 80, 1339-1339.

54 D. Li, M. B. Muller, S. Gilje, R. B. Kaner and G. G. Wallace, Nat. Nanotechnol., 2008, 3, 101-105. 